\title{
Self-Nanoemulsifying Powder of Isotretinoin: Preparation and Characterization
}

\author{
Hitesh Chavda, ${ }^{1}$ Jaimeen Patel, ${ }^{1}$ Gordhan Chavada, ${ }^{1}$ Shruti Dave, ${ }^{2}$ \\ Ankini Patel, ${ }^{2}$ and Chhagan Patel ${ }^{3}$ \\ ${ }^{1}$ Department of Pharmaceutics and Pharmaceutical Technology, Shri Sarvajanik Pharmacy College, \\ Gujarat Technological University, Mehsana, Gujarat 384 001, India \\ ${ }^{2}$ Department of Clinical Pharmacy, Shri Sarvajanik Pharmacy College, Gujarat Technological University, Mehsana, \\ Gujarat 384 001, India \\ ${ }^{3}$ Department of Pharmaceutical Chemistry, Shri Sarvajanik Pharmacy College, Gujarat Technological University, \\ Mehsana, Gujarat 384 001, India \\ Correspondence should be addressed to Hitesh Chavda; hitcvd@yahoo.com
}

Received 8 May 2013; Revised 7 August 2013; Accepted 25 September 2013

Academic Editor: Jürgen Tomas

Copyright (C) 2013 Hitesh Chavda et al. This is an open access article distributed under the Creative Commons Attribution License, which permits unrestricted use, distribution, and reproduction in any medium, provided the original work is properly cited.

In the present investigation an attempt was made to enhance the solubility and dissolution of poorly soluble drug, isotretinoin, by formulating self-nanoemulsifying drug delivery system (SNEDDS). Liquid SNEDDSs were prepared using Transcutol P as oil, Tween 80 as surfactant, and PEG 400 as cosurfactant. Pseudoternary phase diagrams were constructed to identify the efficient selfnanoemulsification region. The formulation with $40 \%$ oil (Transcutol P) and 60\% surfactant: cosurfactant (Tween 80: PEG 400) ratio of $1: 1$ was optimized based on evaluation parameters for droplet size analysis, self-emulsification capacity, zeta potential, and in vitro drug release performance. The optimized system contains mean droplet size of $36.60 \mathrm{~nm}$ and zeta potential $(\zeta)-26.73 \mathrm{mV}$. The optimized formulation Al was adsorbed onto Fujicalin to produce solid SNEDDS, which exhibited good flow properties and preserved the self-emulsification properties of liquid SNEDDS. The differential scanning calorimetry, FT-IR studies of solid SNEDDS revealed transformation of isotretinoin into molecularly dissolved state in the liquid SNEDDS. In vitro dissolution profiles showed that dissolution rate of ISN from solid SNEDDS was significantly greater as compared to pure drug.

\section{Introduction}

Many drug candidates show low water solubility and hence might have a problem of bioavailability, intrasubject or intersubject variability, and lack of dose proportionality [1]. Thus the oral delivery of these low soluble drugs is difficult where dissolution is rate limiting step [2]. The various strategies such as solid dispersions [3], complexation with cyclodextrin [4] and lipid based formulations [5], and self-emulsifying drug delivery systems (SEDDS) [6] have been reported in the literature. Of various strategies reported SEDDS are found to be the prominent approach to improve solubility. SEDDS improve the oral bioavailability of poorly soluble drugs by improving the solubility and maintaining the drug in a dissolved state, in small droplets of oil, all over its transport through the gastrointestinal tract [7]. SEDDS is an isotropic mixture that is comprise of drug, oil, surfactant, and cosurfactant. It readily dispersed in the aqueous environment of the gastrointestinal tract and forms a fine oil-in-water emulsion. If the droplet size of SEDDS is less than $100 \mathrm{~nm}$ it is self-nanoemulsifying (SNEDDS) and for less than $5 \mu \mathrm{m}$ it is self-microemulsifying (SMEDDS) under gentle agitation which improves the oral bioavailability of poorly watersoluble drugs. Compared to ordinary metastable emulsions, SNEDDS shows thermodynamic stability with high solubilization power for lipophilic drugs, as well as it can be filled directly into capsules for convenient oral administration [8]. Norvir (ritonavir), Sandimmune Neoral (cyclosporin), and Fortovase (saquinavir) are SNEDDS based commercialized products [9]. However, there exist a few limitations associated with this delivery system, including stability, interaction with the capsule body, and storage temperature [10]. When the 
product is stored at a lower temperature, there may be some precipitation of the active ingredient and/or the excipients. Therefore the precipitated materials should be dissolved again when warmed to room temperature; otherwise the isotretinoin (ISN) will not be available in a solution form or as fine droplets of emulsion. Moreover it has been suggested that the efficiency of the SEDDS formulation is drug dependent in most instances [11]. Thus, the successful composition of the SEDDS formulation should be carefully explored. To overcome such complications the liquid SEDDS are generally adsorbed onto inert carriers to convent them into solid SEDDS. This approach of solid SEDDS has advantages like stability, facility of manufacturing process, accuracy, and patient compliance. Thus incorporation of liquid SEDDS into solid dosage forms combines the advantages of lipid based drug delivery systems with those of solid dosage forms.

ISN is highly lipophilic vitamin A derivative used in treatment of severe nodular acne. The oral bioavailability of ISN is approximately $25 \%$ due low aqueous solubility. In the current study we developed liquid SNEDDS with Transcutol $\mathrm{P}$ as oil, Tween 80 as surfactant, and PEG 400 as cosurfactant. The prepared liquid SNEDDS was formulated into solid SNEDDS by adsorbing on Fujicalin as a carrier.

\section{Materials and Methods}

2.1. Materials. ISN was purchased from Zeel Pharmaceuticals (Mumbai, India). Transcutol P (highly purified diethylene glycol monoethyl ether), Labrafil M 1944CS (polyoxyethylated oleic glycerides), and medium chain triglycerides such as Lauroglycol, Plurol olieque were kindly provided by Gattefosse (Saint-Priest, France). Captex 355 (glycerol caprylate caprate), Capmul MCM EP (glyceryl mono-dicaprylatel,2,3propanetriol decanoic acid monoester), Captex 200, and Acconon C-80 were obtained as gift samples from ABITEC Corporations (Cleveland, USA). Acrysol El 135 was obtained as gift sample from Corel chemicals (Ahmedabad, India). Fujicalin (spray dried calcium phosphate anhydrous) was generously donated by Fuji chemicals (Toyama-Pref. Japan). Tween 80 (polyoxyethylene sorbitan monooleate), Tween 20 (polyoxyethylene sorbitan monolaurate) were purchased from Finar chemicals (Ahmedabad, India). Polyethylene glycol (PEG) 200 and PEG 400 were purchased from S.D fine chemicals (Mumbai, India). All the excipients and reagents were of analytical grade and double distilled water was freshly prepared whenever required throughout the study.

2.2. Solubility Study. Solubility of ISN was determined in various modified oils, surfactant and cosurfactants. $2 \mathrm{~mL}$ of each component was taken in screw cap vials with excess quantity $(100 \mathrm{mg})$ of ISN. The vials were heated on water bath at $40^{\circ} \mathrm{C}$ to facilitate the solubilization using vortex mixer. The mixtures were agitated on a rotary shaker for $48 \mathrm{hr}$ at $25 \pm 2^{\circ} \mathrm{C}$ for 48 hours. After equilibrium the samples were centrifuged at $6000 \mathrm{rpm}$ for 15 minutes. Supernatant was filtered through membrane filter using $0.45 \mu \mathrm{m}$ filter disk. The solution was filtered and appropriately diluted with methanol and UV absorbance was measured at $344 \mathrm{~nm}$. Concentration of dissolved drug was determined using standard equation [12].

2.3. Selection of Surfactant and Cosurfactant. Briefly, $300 \mathrm{mg}$ of the surfactants was mixed to $300 \mathrm{mg}$ of the selected oily phase. The mixtures were heated at $50^{\circ} \mathrm{C}$ for the homogenization of the components. From that, $50 \mathrm{mg}$ was then diluted with distilled water to $50 \mathrm{~mL}$ in a stopper conical flask. The emulsification efficiency was determined by counting the number of flask inversions required to yield a good homogenous emulsion. Emulsions were kept for $2 \mathrm{~h}$ and their transparency was evaluated at $650 \mathrm{~nm}$ by a double-beam UVvisible spectrophotometer using distilled water as a blank. The screening of the cosurfactant was done on the basis of transparency and ease of emulsification. Mixtures of the selected cosurfactant $(100 \mathrm{mg})$, surfactant $(200 \mathrm{mg})$, and oil $(300 \mathrm{mg})$ were made and evaluated in a similar manner as described in the above section on surfactant [13].

2.4. Ternary Phase Diagram. Surfactant (Tween 80) and cosurfactant (PEG 400) were mixed (S/CoS) in different volume ratios $(1: 1,2: 1,3: 1,1: 2$, and $1: 3)$. For each phase diagram, oil (Transcutol P) and specific S/CoS ratio were mixed vigorously in different volume ratios from 1:9 to 9:1 in different glass vials at room temperature. Ternary phase diagrams were developed using the aqueous titration method. Slow titration with the aqueous phase was done for each combination of oil and S/CoS separately. The quantity of aqueous phase added was speckled to produce a water concentration in the range of $5 \%$ to $95 \%$ of total volume at around 5\% intervals. After each 5\% addition of the aqueous phase to the oil: S/CoS mixture, visual observation was made and recorded [14]. Through visual observation the following categories were assigned:

(1) transparent and easily flowable: oil/water nanoemulsions;

(2) transparent gel: nanoemulsion gel;

(3) milky or cloudy: emulsion;

(4) milky gel: emulgel;

For each S/CoS ratio, a separate phase diagram was constructed using SIGMA PLOT software based on the observations.

2.5. Preparation of Liquid SNEDDS. The surfactant/cosurfactant ratio and oil/S/CoS ratio were selected from the ternary phase diagram. S/CoS $(1: 1)$ and oil to S/CoS ratio of $40: 60-50: 50$ was taken to formulate four batches (Table 1 ). ISN (10 mg) was added in accurately weighed amount of oil into a screw-capped glass vial and heated in a water bath at $40^{\circ} \mathrm{C}$. The surfactant and cosurfactant were added to the oily mixture using pipette and stirred with vortex mixer. The formulations were further sonicated (Ultrasonicator EN30-US, Electroquip, India) for $15 \mathrm{~min}$ and stored at room temperature until further use. 
TABLE 1: Composition of SNEDDS formulations of isotretinoin (\%v/v).

\begin{tabular}{lccc}
\hline \multirow{2}{*}{ Formulation code } & \multicolumn{3}{c}{ Composition (\%v/v) } \\
& Transcutol P & Tween 80 & PEG 400 \\
\hline A1 & 40 & 30 & 30 \\
A2 & 44 & 28 & 28 \\
A3 & 46 & 27 & 27 \\
A4 & 50 & 25 & 25 \\
\hline
\end{tabular}

\subsection{Characterization of Liquid SNEDDS}

2.6.1. Drug Content. ISN from SNEDDS formulations were extracted in methanol using sonication technique. The solutions were filtered, using Whatman filter paper of $0.45 \mu \mathrm{m}$ pore size. The methanolic extracts were analyzed for the ISN content spectrophotometrically (UV-1800, Shimadzu, Japan) at $344 \mathrm{~nm}$ using standard curve.

2.6.2. Emulsification Time. Self-emulsifying properties of SNEDDS formulations were determined by visual assessment. The USP XXIV type II dissolution apparatus was used to evaluate the efficiency of self-emulsification of different formulations. One gram of each formulation was added drop wise into $500 \mathrm{~mL}$ of distilled water maintained at $37^{\circ} \mathrm{C}$ with gentle agitation condition provided by rotating paddle at $50 \mathrm{rpm}$. Time required for the complete disappearance of SNEDDS was recorded [12].

2.6.3. Transmittance. Percentage transmittance of the prepared emulsion formulations were determined spectrophotometrically using Shimadzu UV-visible spectrophotometer. One $\mathrm{mL}$ of the formulations was diluted 100 times using methanol and analyzed at $344 \mathrm{~nm}$ using methanol as blank [15].

2.6.4. Droplet Size Analysis. The droplet size and polydispersity index of the formulations were determined by Zetasizer Nano ZS (Malvern Instruments, UK). Each formulation was diluted with distilled water before analysis. Size analysis was monitored at $25^{\circ} \mathrm{C}$ at $90^{\circ}$ angle.

2.6.5. Zeta Potential Measurement. Zeta potential of the formulations was measured by using Malvern Zetasizer Nano ZS (Malvern Instruments, UK). Zetasizer measures the potential ranged from -120 to $120 \mathrm{mV}$. For zeta potential measurement $2 \mathrm{~g}$ of each formulation was diluted with water $(100 \mathrm{~mL})$.

2.7. Preparation of Solid SNEDDS. The solid self-emulsifying powder was prepared for liquid SNEDDS (A1). Fujicalin was used as solid adsorbent carriers to formulate solid SNEDDS. Briefly the fixed aliquot of liquid SNEDDS was added and mixed vigorously with adsorbent in the mortar until it became free flowing. The powder was then passed through 60 mesh sieve $(250 \mu \mathrm{m})$ to get uniform particle size. The powder samples were stored in a desiccator until further evaluation.

\subsection{Characterization of Solid SNEDDS}

2.8.1. Powder Flow Properties. The angle of repose of powder blend was determined by funnel method. The accurately weighed powder blend was taken in funnel. The height of funnel was adjusted in such a ways that the tip of funnel just touched the apex of powder blend. The powder mixture was allowed to flow through the funnel freely onto the surface. The diameter of the powder cone was measured and angle of repose $(\theta)$ was calculated using the equation, $\theta=\tan ^{-1} h / r$, where $h$ and $r$ are the height and radius of powder cone. Carr's Index was determined to check the compressibility of solid SNEDDS powder which is helpful to understand the maximum fill volume in capsule. Carr's index can be calculated using equation, Carr's Index (\%) $=[($ Tapped density - Bulk density $) \times 100] /$ Tapped density. Hausner's ratio was calculated using the equation, Hausner's ratio $=$ Tapped density/Bulk density.

2.8.2. Solid State Characterization. The physical state of ISN in solid SNEDDS was determined by differential scanning calorimetry studies and FT-IR studies [16, 17]. The DSC thermograms of the ISN and solid SNEDDS were recorded using differential scanning calorimeter (Shimadzu, Japan). 2$3 \mathrm{mg}$ sample was taken in DSC aluminum cell and studied at temperature range of 30 to $300^{\circ} \mathrm{C}$ at a nitrogen flow rate of $40-50 \mathrm{~mL} / \mathrm{min}$. The infrared spectrum of pure ISN and ISN loaded solid SNEDDS was recorded on a Fourier transform infrared spectrophotometer (Shimadzu, Japan) in the range of $4000-400 \mathrm{~cm}^{-1}$.

2.8.3. Reconstitution Properties. The droplet size, polydispersity index, and zeta potential of the solid SNEDDS were determined by Zetasizer Nano ZS (Malvern Instruments, UK). Formulation was diluted with distilled water before analysis. Size analysis was monitored at $25^{\circ} \mathrm{C}$ at $90^{\circ}$ angle.

2.9. In Vitro Drug Release Studies. The in vitro drug release of ISN from the optimized SNEDDS and solid SNEDDS was performed using USP dissolution Apparatus II (TDT08L, Electrolab, Mumbai, India). Hard gelatin capsules, size " 00 " filled with preconcentrate (equivalent to $10 \mathrm{mg}$ ISN) were put into each of $900 \mathrm{~mL}$ dibasic phosphate buffer $\mathrm{pH}$ 7.8 , at $37 \pm 0.5^{\circ} \mathrm{C}$ with a $50 \mathrm{rpm}$ rotating speed. Samples $(10 \mathrm{~mL})$ were withdrawn at regular time intervals $(5,10$, $20,30,40,50$, and $60 \mathrm{~min}$ ) and filtered using a $0.45 \mu \mathrm{m}$ filter. The same volume of the dissolution medium was added to maintain the volume constant and maintain sink condition. The drug content of the samples was analyzed using UV visible spectrophotometric method at $344 \mathrm{~nm}$. All measurements were done in triplicate.

2.10. Ex Vivo Intestinal Permeability Studies. Male Wister rats $(250-300 \mathrm{~g})$ were sacrificed by $\mathrm{CO}_{2}$ inhalation method. Intestine isolated and cleaned properly. One milliliter of ISN solid SNEDDS and plain drug suspension sample $(10 \mathrm{mg} / \mathrm{mL})$ was filled into the intestine individually which was tied at both the ends. The tissue was placed in an organ bath 


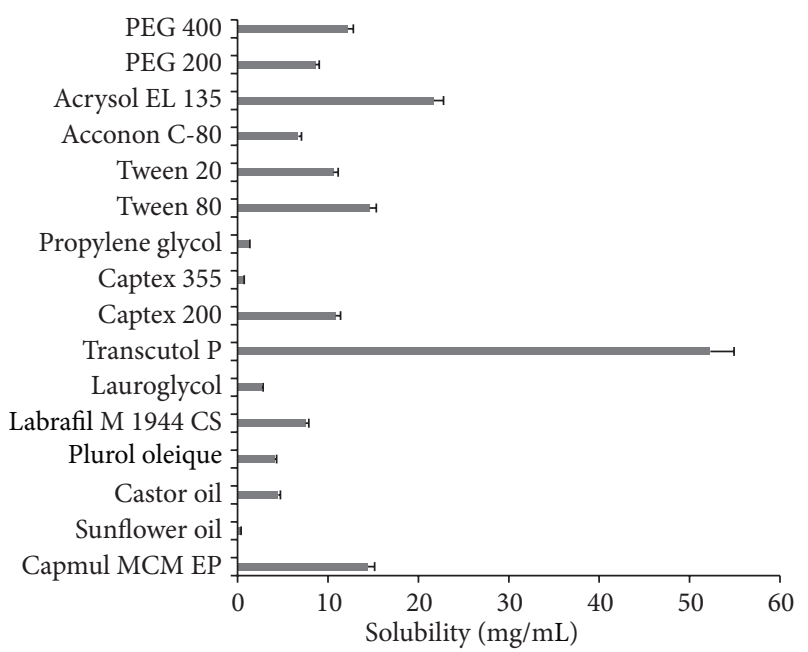

FIGURE 1: Solubility studies of isotretinoin in various excipients (Mean $\pm \mathrm{SD}, n=3$ ).

TABLE 2: Emulsification efficiency of various surfactants and cosurfactants (Means, $n=3$ ).

\begin{tabular}{lcc}
\hline Surfactants/cosurfactants & Transparency (\%) & No. of inversions \\
\hline Tween 80 & 98.62 & 5 \\
Tween 20 & 98.1 & 15 \\
Plurol Oleique & 90.6 & 25 \\
PEG 200 & 98.1 & 35 \\
PEG 400 & 99.5 & 20 \\
Lauroglycol & 95.1 & 20 \\
Capmul MCM EP & 88.3 & 40 \\
\hline
\end{tabular}

with continuous aeration at $37^{\circ} \mathrm{C}$. The receptor compartment (organ tube) was filled with $30 \mathrm{~mL}$ of dibasic potassium phosphate buffer $\mathrm{pH}$ 7.8. At predetermined time intervals, samples were withdrawn from the receptor compartment. Fresh buffer was used to replenish the receptor compartment. The samples were analyzed spectrophotometrically for the content of ISN at $344 \mathrm{~nm}$. The percent diffusion was calculated and plotted against time. All the experiments were done in triplicate.

\section{Results and Discussion}

3.1. Solubility Studies. The results of solubility of ISN in various oils, surfactants, and cosurfactants screened were shown in Figure 1. The components in formulation of SNEDDS were selected to have maximum solubility of drug along with good miscibility with each other and to produce a stable formulation [16]. The results suggest that ISN is highly soluble in Transcutol P $(52.3 \pm 1.01 \mathrm{mg} / \mathrm{mL})$, Tween $80(14.6 \pm$ $2.08 \mathrm{mg} / \mathrm{mL})$, and PEG $400(12.2 \pm 0.95 \mathrm{mg} / \mathrm{mL})$. Based on the solubility results the SNEDDS formulations were developed employing varying concentrations of Transcutol P (40-50\%), Tween 80 (25-30\%), and PEG 400 (25-30\%).

3.2. Selection of Surfactant and Cosurfactant. In this study, the four surfactants (Tween 80, Tween 20, Lauroglycol,

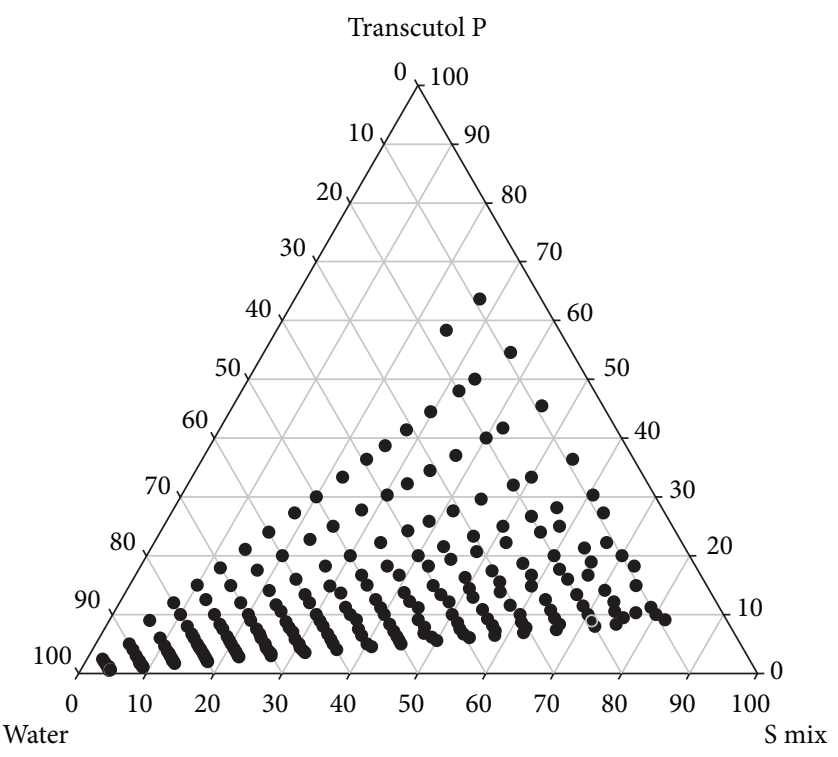

FIGURE 2: Ternary phase diagram of SNEDDS between Transcutol P, Tween 80/PEG 400 (1:1) and Water (dotted area represents the region of stable emulsions).

and Capmul MCM EP) were compared. Well-formulated SNEDDS dispersed within seconds under gentle stirring which ultimately depends on the surfactant emulsification ability. Results showed that the oily phase Transcutol P exhibited the highest emulsification efficiency with Tween 80 (transparency in \%: 98.62, 5 flask inversions (5s)) for the homogenous emulsion formation. On the other side, Transcutol $\mathrm{P}$ showed poor emulsification properties with other surfactants engaged, requiring large number of flask inversions (Table 2). The results suggested the use of Transcutol $\mathrm{P}$ as an oily phase with Tween 80 as a surfactant for further study. Addition of a cosurfactant to the surfactantcontaining formulation improved the dispersibility and drug absorption from the formulation. In the present investigation, three cosurfactants, namely, PEG 200, PEG 400, and Plurol Oleique, were compared. As showed in Table 2, Transcutol $\mathrm{P}$ exhibited good emulsification with all cosurfactants, with PEG 400 showing the maximum transmittance (99.5\%). Herein, the solubility of the drug in different cosurfactants may umpire the final selection. Results of the solubility study demonstrated in Figure 1 showed a higher solubility in PEG 400 .

3.3. Ternary Phase Diagram. A ternary phase diagram was investigated to choose the proper concentration of excipients, that is, oil proportion and optimum $S / C o S$ ratio in the formulation to produce emulsions with good stability (the dotted area in Figure 2). All the emulsions were stable at zero time and this may be due to higher HLB value of Tween 80 (HLB15) and higher solubilizing capacity of PEG 400. After observing clarity, stability after $48 \mathrm{~h}$, it was noted that formulations with $S / C o S$ ratio of $1: 1$ and oil/S/CoS ratio of $50: 50$ produced stable emulsions, whereas the resultant emulsions of formulations with $\mathrm{S} / \mathrm{CoS}$ ratios of $2: 1,3: 1$, 
TABLE 3: Visual observations for ternary phase diagram construction using different S/CoS ratio.

\begin{tabular}{|c|c|c|c|c|c|}
\hline \multirow{2}{*}{ Oil: $\mathrm{S} / \mathrm{CoS}$} & \multicolumn{5}{|c|}{$\%$ of water at which turbidity observed for different $\mathrm{S} / \mathrm{CoS}$ ratio } \\
\hline & $1: 1$ & $2: 1$ & $3: 1$ & $1: 2$ & $1: 3$ \\
\hline $10: 90$ & NT & NT & NT & NT & NT \\
\hline $20: 80$ & NT & NT & NT & NT & NT \\
\hline $30: 70$ & NT & NT & 80 & NT & NT \\
\hline $40: 60$ & NT & NT & 75 & NT & 85 \\
\hline $50: 50$ & NT & 85 & 44 & 70 & 64 \\
\hline $60: 40$ & 90 & 35 & 17 & 65 & 60 \\
\hline $70: 30$ & 20 & 9 & 9 & 9 & 9 \\
\hline $80: 20$ & 9 & 9 & 9 & 9 & 9 \\
\hline $90: 10$ & 9 & 9 & 9 & 9 & 9 \\
\hline
\end{tabular}

${ }^{*} \mathrm{NT}$ : no turbidity.

$1: 2$, and $1: 3$ showed phase separation and precipitation. Results of the Ternary Phase Diagram also showed that as the concentration of the oil was increase the turbidity of formulation was increase (results shown in Table 3). This may be attributed to increased interfacial tension between oil and aqueous phase due to insufficient concentration of surfactant system.

3.4. Characterization of Liquid SNEDDS. Table 4 shows drug content, emulsification time, and transmittance. Despite of difference in composition, the drug content of formulations A1 to A4 was found in range of 98.71-99.56\%. Batch A1 was found to have $99.56 \%$ ISN. The efficiency of selfemulsification could be determined primarily by the rate of emulsification. The results suggest that the formulations containing $40 \%$ oil content showed the emulsification time of less than $16 \mathrm{~s}$. However with increase of oil proportion to $50 \%$ the emulsification time was increased. These visual observations indicated that the higher the proportion of surfactant system, the higher the rate of self-emulsification. No marginal difference was observed among the percentage transmittance of formulations and formulation A1 was found to have the highest percentage of transmittance. A value of percentage transmittance closer to $100 \%$ signified that all of the formulations were clear and transparent. Besides signifying clarity of the formulation, a percentage transmittance closer to $100 \%$ also indicates that the size of the droplets in the formulation is in the nanometer range. This in turn indicates that the drug in the formulation has a large surface area for release. A1 formulation shows high drug content, transmittance, and lowest emulsification time compared to other formulations.

The droplet size of an emulsion is a vital factor in self-emulsification characteristics because it determines the rate and extent of drug release as well as absorption [18]. Droplet size of batches, polydispersity index (PDI), and $\zeta$ potential are shown in Table 5. PDI less than 0.5 indicates a homogenous distribution, while a greater than 0.5 indicates a higher heterogeneous dispersion. Here as we increased surfactant/cosurfactant proportion there was a decrease in droplet size. All formulations show size less than $65 \mathrm{~nm}$;
TABLE 4: Drug content, emulsification time, and transmittance of the developed liquid SNEDDS formulations (Mean \pm SD, $n=3$ ).

\begin{tabular}{lccc}
\hline Formulations Drug content & $\begin{array}{c}\text { Emulsification time } \\
(\mathrm{sec})\end{array}$ & $\begin{array}{c}\text { Transmittance } \\
(\%)\end{array}$ \\
\hline A1 & $99.56 \pm 0.24$ & $15 \pm 2$ & $99.79 \pm 0.01$ \\
A2 & $99.49 \pm 0.41$ & $18 \pm 2$ & $99.68 \pm 0.03$ \\
A3 & $98.71 \pm 0.81$ & $26 \pm 3$ & $99.44 \pm 0.01$ \\
A4 & $99.32 \pm 0.09$ & $36 \pm 2$ & $99.13 \pm 0.04$ \\
\hline
\end{tabular}

TABLE 5: Droplet size, polydispersibility index (PDI), and $\zeta$-potential of the developed SNEDDS formulations.

\begin{tabular}{lccc}
\hline Formulations & Droplet size $(\mathrm{nm})$ & PDI & $\zeta$-Potential $(\mathrm{mV})$ \\
\hline A1 & 29.53 & 0.343 & -28.50 \\
A2 & 40.25 & 0.310 & -23.89 \\
A3 & 47.79 & 0.623 & -26.12 \\
A4 & 64.71 & 0.263 & -20.19 \\
\hline
\end{tabular}

hence the system is in nanosize range. However for formulation A3 PDI is greater than 0.5, which shows heterogeneous dispersion of droplets in SNEDDS supported by Figure 3. Formulations A4 and A1 showed good droplet size over A2. As shown in the figure the last two peaks for A1 and A2 are not in nanosize but having low intensity compared to first peak. So majority of droplets are in nanorange. For formulation A4 there is only two peaks and the third is absent which is clearly indicating nanosize of droplets.

$\zeta$-Potential of batches A1 to A4 is shown in Table 5. Since, $\zeta$-potential governs the degree of repulsion between adjacent, similarly charged, and dispersed droplets, it shows practical application in the emulsion stability. If the $\zeta$-potential is reduced below a certain value (which depends on a particular system being used), the attractive forces exceed the repulsive forces, and the globules come together leading to flocculation. The zeta potential of the formulations was found in the range from -20.19 to $-28.50 \mathrm{mV}$. In general, the zeta potential value of $\pm 30 \mathrm{mV}$ is sufficient for the stability of an emulsion. Batch A1 has zeta potential $-28.50 \mathrm{mV}$ so it can be considered as the most stable formulation. 

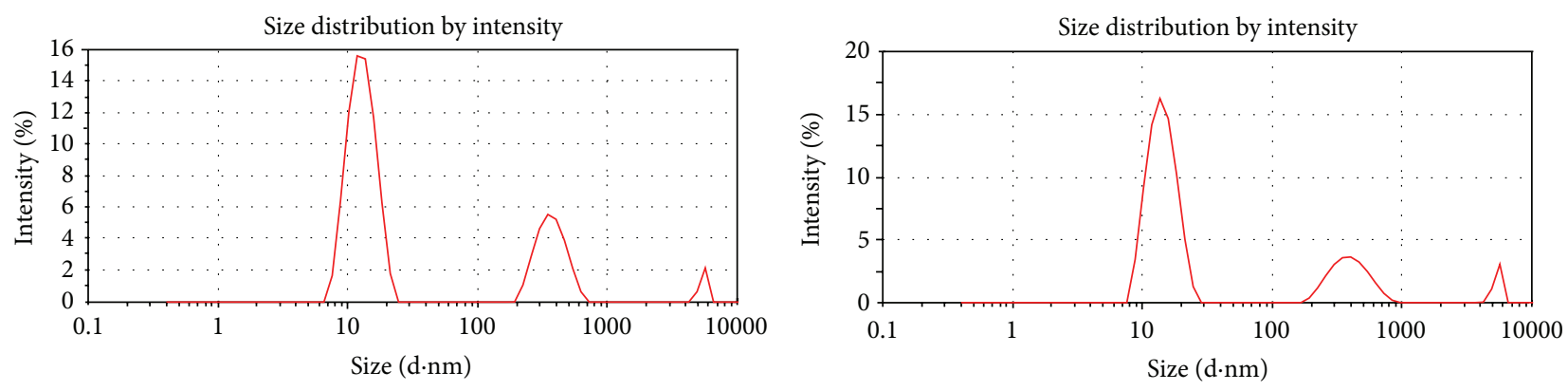

$-\mathrm{A} 1$

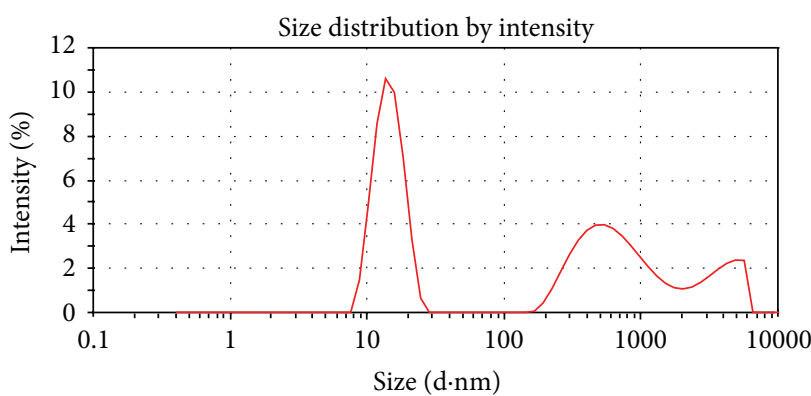

$-\mathrm{A} 3$

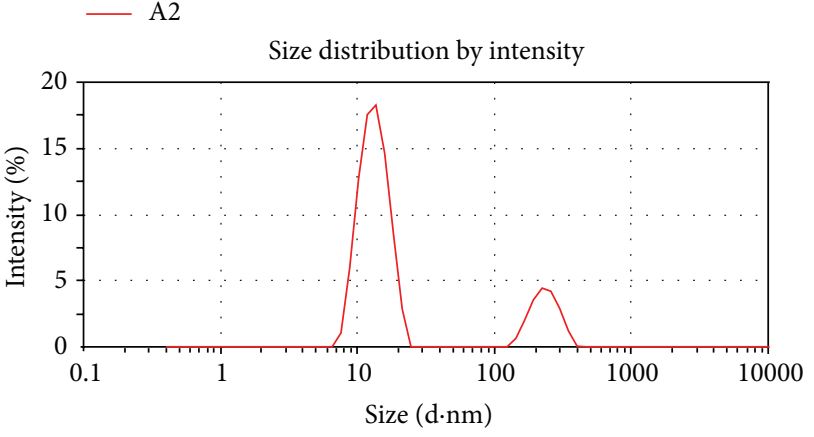

- A4

Figure 3: Droplet size distribution in A1 to A4 batch.

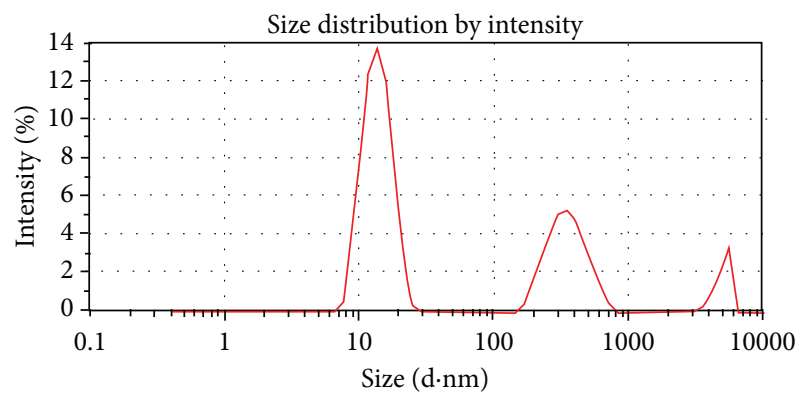

FIGURE 4: Droplet size distribution in solid SNEDDS.

From Tables 4 and 5 it is clear that formulation Al is more suitable for SNEDDS and so further evaluated for concerned properties and parameters.

\subsection{Characterization of Solid SNEDDS}

Powder Flow Properties. Solid SNEDDS was prepared to overcome the disadvantages associated with liquid SNEDDS. Hence to increase the stability and patient compliance optimized formulation Al was adsorbed onto Fujicalin. The amount of Fujicalin adsorbed to produce the free flowing powder was $1.08 \mathrm{~g} / \mathrm{mL}$ of SNEDDS formulation. This low amount of Fujicalin required to produce free flowing characteristics may be due to the larger adsorption capacity $110 \mathrm{~mL} / 100 \mathrm{~g}$ of Fujicalin. The solid SNEDDS had an angle of repose value of $28.15^{\circ}$, Hausner's ratio of 1.13 , and Carr's index of 22.34 which indicated good flow and compression characteristic that is helpful in capsule filling. As shown in

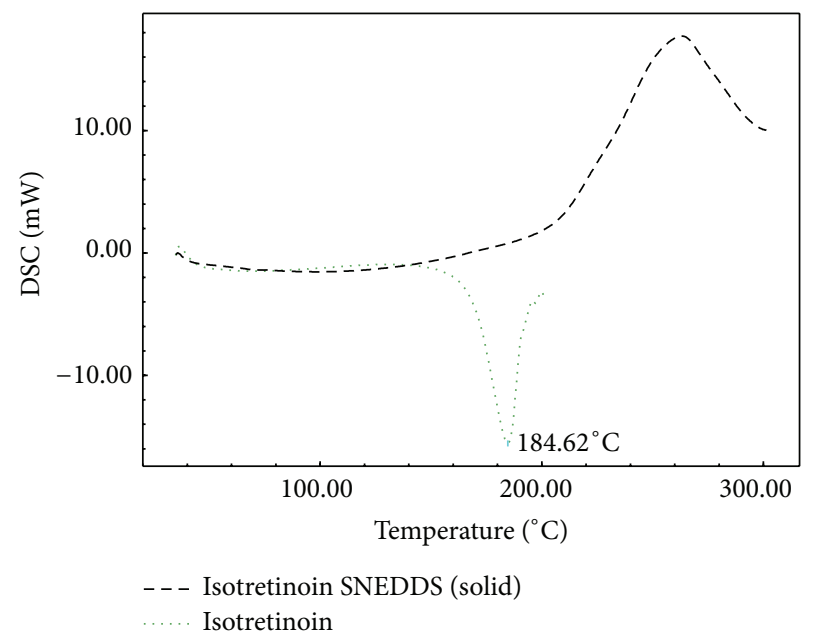

FIGURE 5: DSC thermograms of pure isotretinoin and solid SNEDDS.

Figure 4 droplet size distribution broaden is compared to liquid SNEDDS. This may be due to the adsorption of liquid SNEDDS on Fujicalin.

Solid State Characterization. The physical state of ISN in the solid SNEDDS was investigated since it would have an important influence on the in vitro and in vivo release characteristics. DSC curves of pure ISN and solid SNEDDS are shown in Figure 5. Pure ISN showed a sharp endothermic peak at about $184.62^{\circ} \mathrm{C}$, which is correspondence to its melting point $173-178^{\circ} \mathrm{C}$ [19]. No obvious peak of the drug 


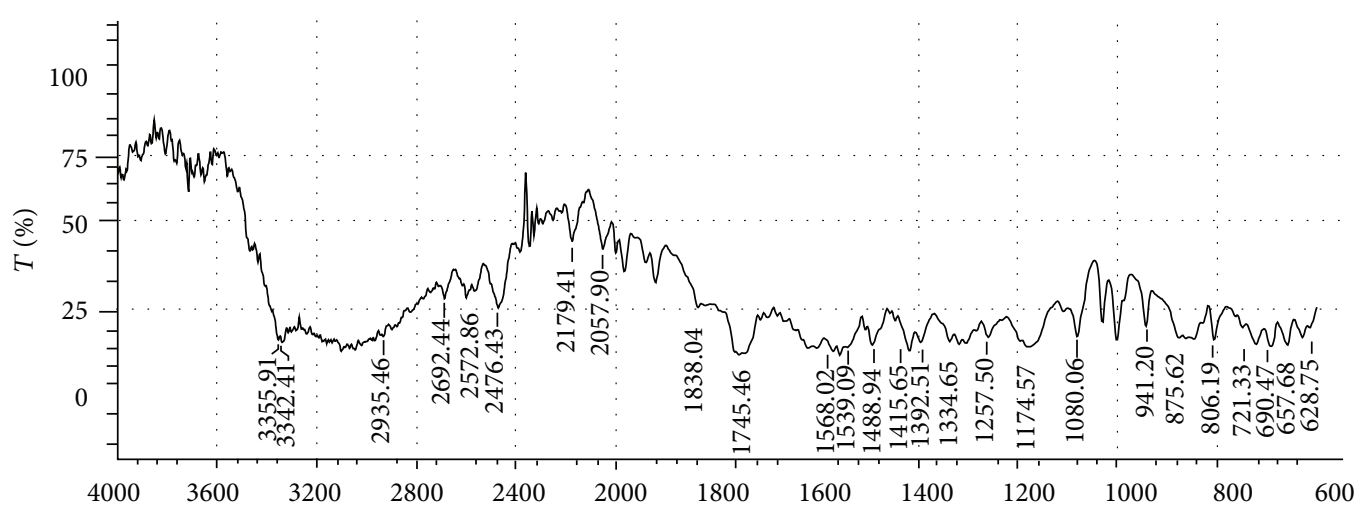

(a) FT-IR spectrum of isotretinoin

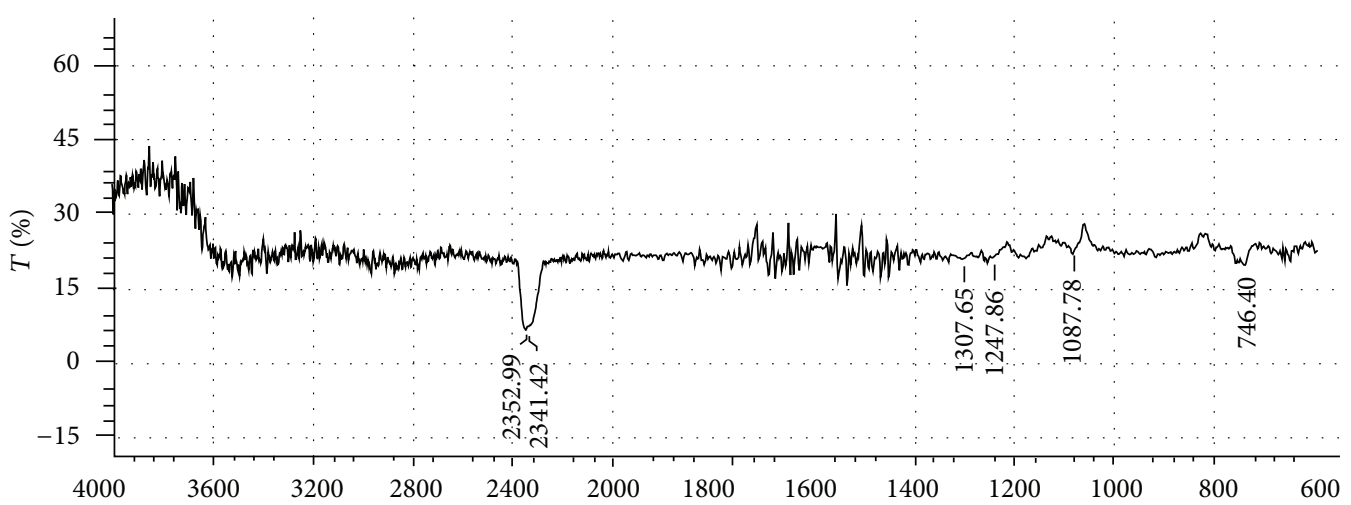

(b) FT-IR spectrum of solid SNEDDS

FIGURE 6: FT-IR spectrum of pure isotretinoin and solid SNEDDS.

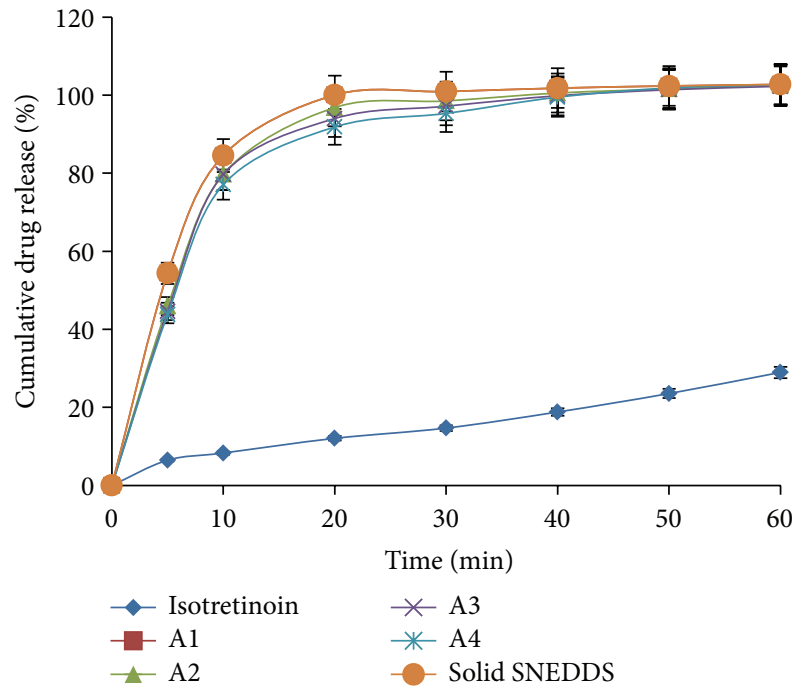

FIGURE 7: In vitro release profile of liquid SNEDDS formulations and pure isotretinoin (Mean $\pm \mathrm{SD}, n=3$ ).

was found in the solid SNEDDS of ISN, indicating that the drug must be present in molecularly dissolved state in solid SNEDDS. FT-IR spectra of pure ISN and solid SNEDDS are shown in Figure 6. Pure drug showed characteristics band at
TABLE 6: Droplet size, polydispersibility index (PDI), $\zeta$-potential, and emulsification time of the optimized liquid SNEDDS (A1) and solid SNEDDS.

\begin{tabular}{lcccc}
\hline Formulation & Size $(\mathrm{nm})$ & PDI & $\begin{array}{c}\text { Zeta potential } \\
(\mathrm{mV})\end{array}$ & $\begin{array}{c}\text { Emulsification } \\
\text { time }(\mathrm{sec})\end{array}$ \\
\hline A1 & 29.53 & 0.343 & -28.50 & $15 \pm 2$ \\
Solid SNEDDS & 36.60 & 0.411 & -26.73 & $21 \pm 2$ \\
\hline
\end{tabular}

$1745 \mathrm{~cm}^{-1}(\mathrm{C}=\mathrm{O}), 3325 \mathrm{~cm}^{-1}(\mathrm{O}-\mathrm{H}), 1568 \mathrm{~cm}^{-1}(\mathrm{C}=\mathrm{C})$, and $2935 \mathrm{~cm}^{-1}(\mathrm{C}-\mathrm{H})$. None of the characteristic bands for ISN was observed in the solid SNEDDS; thus it is confirmed that drug is in molecularly dissolved state and correlates the DSC data.

Reconstitution Properties. The z-average diameter and polydispersibility index of the solid and liquid SNEDDS are presented in Table 6 . The $\mathrm{z}$-average droplet sizes of both systems were less than $40 \mathrm{~nm}$ (Figure 4). And there is no significant change in zeta potential of both systems. The emulsion droplet size distribution and zeta potential in liquid and solid SNEDDS confirmed the self-emulsification nature of the solid SNEDDS.

3.6. In Vitro Drug Release Studies. Figure 7 shows in vitro dissolution profiles for plain ISN and liquid and solid 


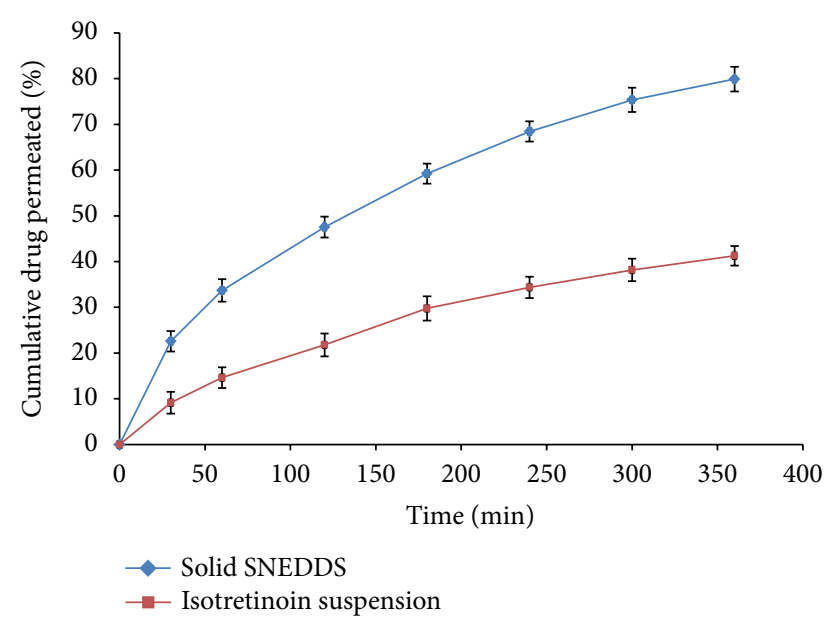

Figure 8: Ex vivo permeation profile of solid SNEDDS and isotretinoin suspension (mean $\pm \mathrm{SD}, n=3$ ).

SNEDDS formulations performed in dibasic potassium phosphate buffer $\mathrm{pH}$ 7.8. There was no marginal difference in drug release of four liquid SNEDDS and solid SNEDDS formulations. All SNEDDS formulations showed 100\% drug release, while plain ISN exhibited only $14.2 \%$ within 20 minute. These might be due to very short emulsification time of just 40 second. The drug release studies were continued for 1 hour to observe the variation/occurrence of precipitation over a time. Drug release profile of solid SNEDDS in buffer solution showed that the formulation had higher drug release profile than the ISN powder, ensuring that the solid SNEDDS preserved the improvement of in vitro dissolution of liquid SNEDDS. The cumulative percentage drug release from solid SNEDDS was found to be $102.83 \pm 1.32 \%$ within $60 \mathrm{~min}$ and was significantly higher than the pure drug $(14.1 \pm 1.1 \%)$.

3.7. Ex Vivo Intestinal Permeability Studies. The results of the ex vivo intestinal permeability study are shown in Figure 8. After $6 \mathrm{~h}$ of diffusion, $79.89 \%$ of the drug was diffused from the solid SNEDDS, while from plain drug suspension the diffusion was found to be $41.27 \%$. Thus, the amount of the drug diffused through the biological membrane has doubled when it was given in the form of a SNEDDS. The enhanced diffusion from the solid SNEDDS may be due to the large specific surface area of the droplets and improved permeation of the ISN because of the presence of surfactant, which reduces the interfacial tension.

\section{Conclusion}

Solid SNEDDS formulation containing ISN significantly improved the dissolution rate and drug diffusion across the intestinal membrane compared to plain ISN suspension. These observations reflect that SNEDDS seems to be a promising approach, which can provide an effective solution to the problem of formulating poorly water soluble drugs with poor systemic bioavailability.

\section{Acknowledgments}

The authors are thankful to ABITEC Corporation (Cleveland, USA), Gattefossé (Saint-Priest, France), Fuji chemical Industry Co., Ltd (Toyama-Pref. Japan), and Corel Pharma Chem (Gujarat, India) for providing generous gift samples.

\section{References}

[1] C. Lipinski, "Poor aqueous solubility-an industry wide problem in drug discovery," American Pharmaceutical Review, vol. 5, no. 3, pp. 82-85, 2002.

[2] A. M. Palmer, "New horizons in drug metabolism, pharmacokinetics and drug discovery," Drug News and Perspectives, vol. 16, no. 1, pp. 57-62, 2003.

[3] I. Weuts, D. Kempen, A. Decorte et al., "Phase behaviour analysis of solid dispersions of loperamide and two structurally related compounds with the polymers PVP-K30 and PVPVA64," European Journal of Pharmaceutical Sciences, vol. 22, no. 5, pp. 375-385, 2004.

[4] H. O. Ammar, H. A. Salama, M. Ghorab, and A. A. Mahmoud, "Implication of inclusion complexation of glimepiride in cyclodextrin-polymer systems on its dissolution, stability and therapeutic efficacy," International Journal of Pharmaceutics, vol. 320, no. 1-2, pp. 53-57, 2006.

[5] J. M. Odeberg, P. Kaufmann, K. Kroon, and P. Höglund, "Lipid drug delivery and rational formulation design for lipophilic drugs with low oral bioavailability, applied to cyclosporine," European Journal of Pharmaceutical Sciences, vol. 20, no. 4-5, pp. 375-382, 2003.

[6] P. Balakrishnan, B. Lee, D. H. Oh et al., "Enhanced oral bioavailability of Coenzyme Q10 by self-emulsifying drug delivery systems," International Journal of Pharmaceutics, vol. 374, no. 12, pp. 66-72, 2009.

[7] C. W. Pouton, "Lipid formulations for oral administration of drugs: non-emulsifying, self-emulsifying and "selfmicroemulsifying" drug delivery systems," European Journal of Pharmaceutical Sciences, vol. 11, no. 2, pp. 93-98, 2000.

[8] Y. Zhaoa, C. Wanga, A. H. L. Chowb et al., "Self-nanoemulsifying drug delivery system (SNEDDS) for oral delivery of Zedoary essential oil: formulation and bioavailability studies," International Journal of Pharmaceutics, vol. 383, pp. 170-177, 2010.

[9] R. N. Gursoy and S. Benita, "Self-emulsifying drug delivery systems (SEDDS) for improved oral delivery of lipophilic drugs," Biomedicine and Pharmacotherapy, vol. 58, pp. 173-182, 2004.

[10] C. G. Wilson and B. O. Mahony, "The behavior of fats and oils in the upper G.I. Tract," Gattefossé Bulletin Technique, vol. 90, pp. 13-18, 1997.

[11] Y. Chen, G. Li, X. Wu et al., "Self-microemulsifying drug delivery system (SMEDDS) of vinpocetine: formulation development and in vivo assessment," Biological and Pharmaceutical Bulletin, vol. 1, pp. 118-125, 2008.

[12] V. R. Kallakunta, S. Bandari, and R. Jukanti, "Oral self emulsifying powder of lercanidipine hydrochloride: formulation and evaluation," Powder Technology, vol. 221, pp. 375-382, 2012.

[13] J. Patel, G. Kevin, A. Patel, M. Raval, and N. Sheth, "Design and development of a self-nanoemulsifying drug delivery system for telmisartan for oral drug delivery," International Journal of Pharmaceutical Investigation, vol. 1, no. 2, pp. 112-118, 2011. 
[14] S. Agrawal, T. Giri, D. Tripathi, and A. Alaxander, "A review on novel therapeutic strategies for the enhancement of solubility for hydrophobic drugs through lipid and surfactant based self micro emulsifying drug delivery system: a novel approach," American Journal of Drug Discovery and Development, vol. 2, no. 4, pp. 143-183, 2012.

[15] V. Bali, M. Ali, and J. Ali, "Nanocarrier for the enhanced bioavailability of a cardiovascular agent: in vitro, pharmacodynamic, pharmacokinetic and stability assessment," International Journal of Pharmaceutics, vol. 403, no. 1-2, pp. 46-56, 2011.

[16] P. Balakrishnan, B. Lee, D. H. Oh et al., "Enhanced oral bioavailability of dexibuprofen by a novel solid Self-emulsifying drug delivery system (SEDDS)," European Journal of Pharmaceutics and Biopharmaceutics, vol. 72, no. 3, pp. 539-545, 2009.

[17] M. Milović, J. Djuriš, L. Djekić, D. Vasiljević, and S. Ibrić, “Characterization and evaluation of solid self-microemulsifying drug delivery systems with porous carriers as systems for improved carbamazepine release," International Journal of Pharmaceutics, vol. 436, pp. 58-65, 2012.

[18] P. P. Constantinides, J.-P. Scalart, C. Lancaster et al., "Formulation and intestinal absorption enhancement evaluation of water-in-oil microemulsions incorporating medium-chain glycerides," Pharmaceutical Research, vol. 11, no. 10, pp. 13851390, 1994.

[19] "Isotretinoin 13-cis-Retinoic acid," 2013, (4759-48-2) MSDS, Chemical Book, http://www.chemicalbook.com/ProductMSDS DetailCB6222631_EN.htm. 

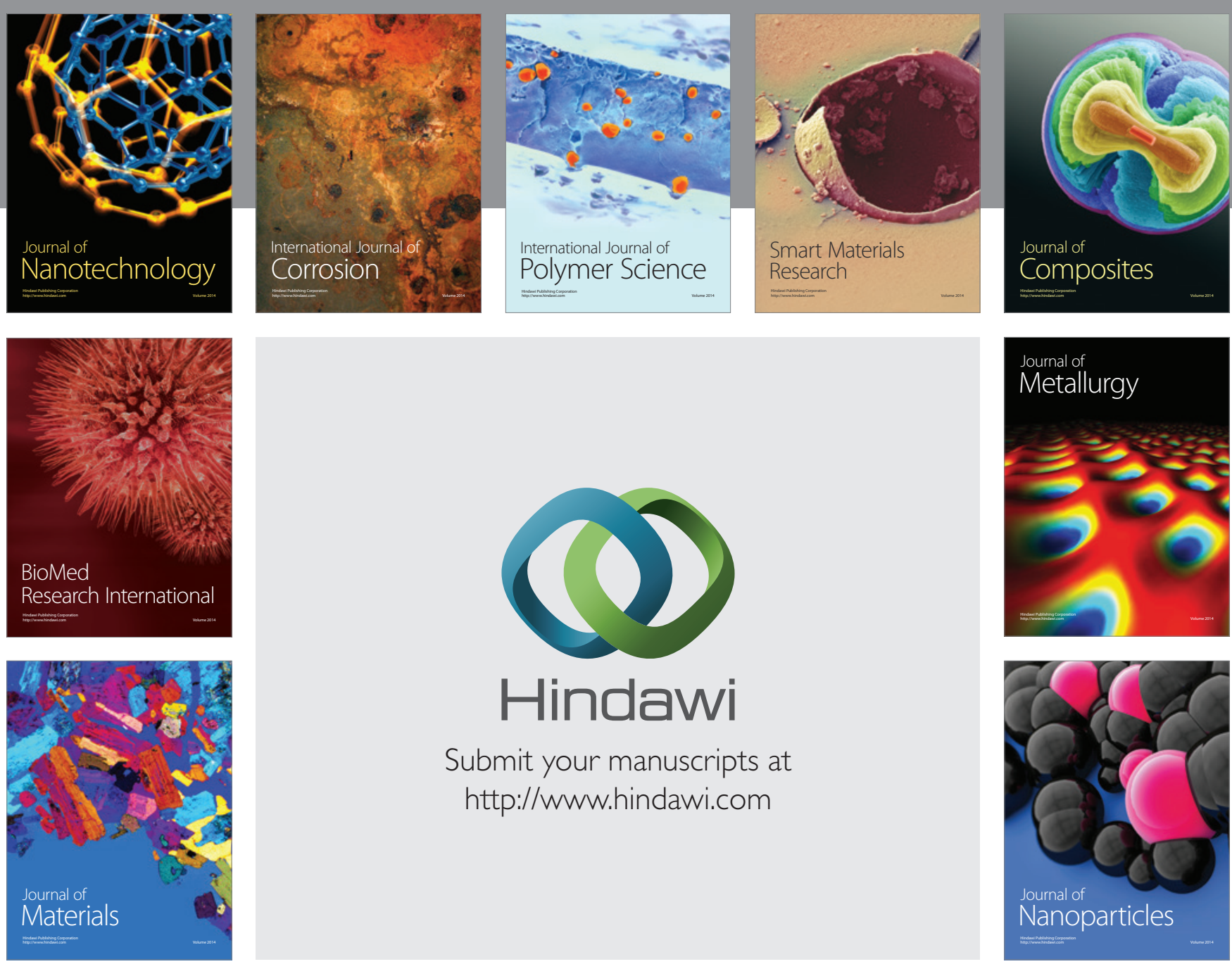

Submit your manuscripts at http://www.hindawi.com
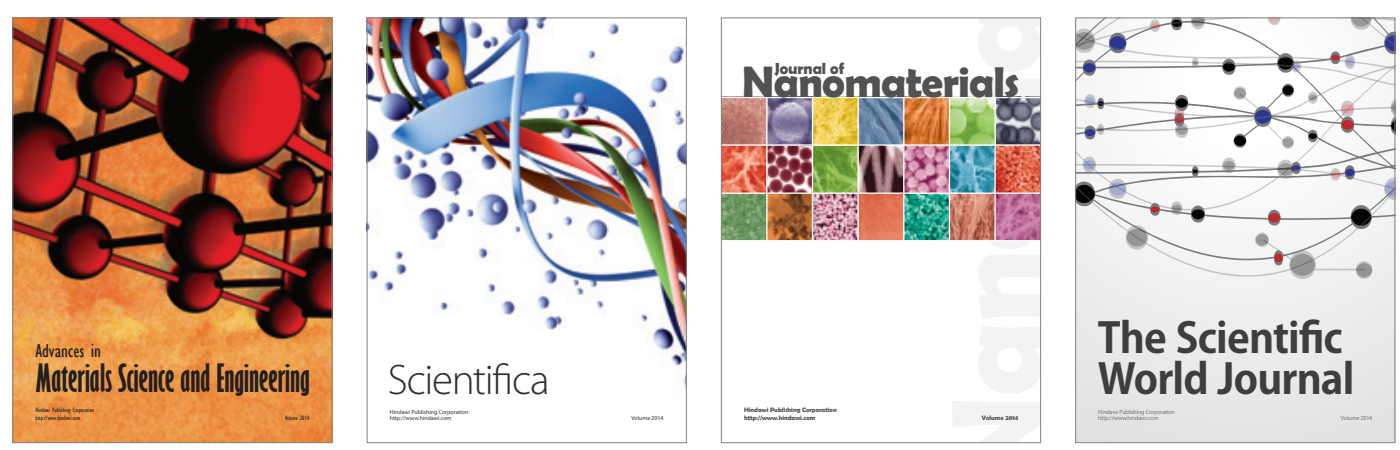

\section{The Scientific World Journal}
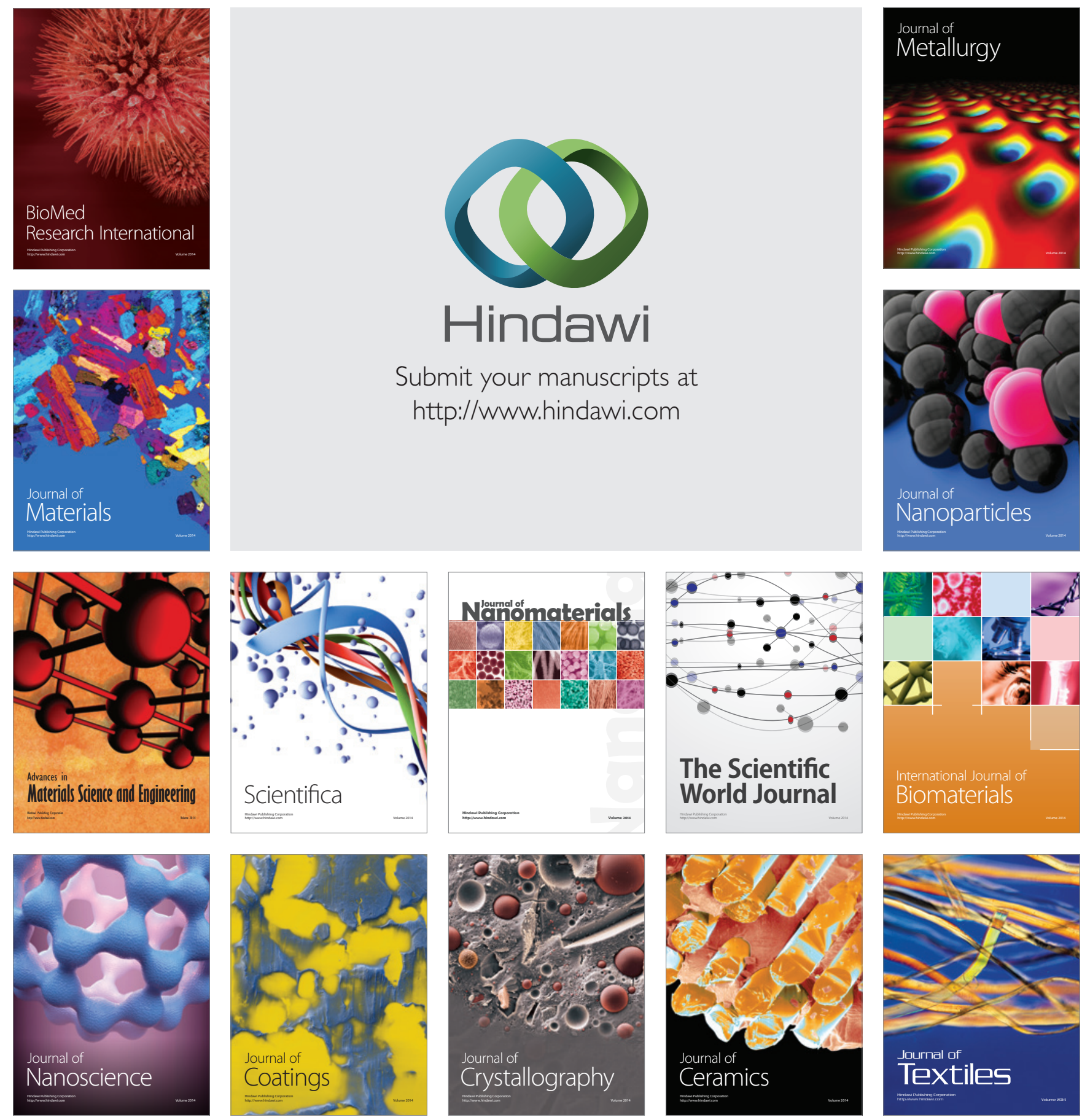